\title{
Cor/log BAN BT a Wearable Battery Powered mHealth Data Logger and Telemetry Unit for Multiple Vital Sign Monitoring
}

\author{
Thomas Hilbel ${ }^{1,2}$, Sven Feilner ${ }^{3}$, Matthias Struck ${ }^{3}$, Christian Hofmann ${ }^{3}$, Andreas Heinig ${ }^{4}$, \\ Hugo A Katus ${ }^{1}$ \\ ${ }^{1}$ Division of Cardiology, University Hospital, Heidelberg, Germany \\ ${ }^{2}$ University of Applied Sciences, Gelsenkirchen, Germany \\ ${ }^{3}$ Fraunhofer Institute for Integrated Circuits IIS, Erlangen, Germany \\ ${ }^{4}$ Fraunhofer Institute for Photonic Microsystems, Dresden, Germany
}

\begin{abstract}
The wireless data logger system "Cor/log ${ }^{\circledR} B A N$ BT" (CL) allows seamless 24/7 monitoring of relevant vital sign parameters. CL covers the entire period of acute point of care inside the hospital and the recovery period, when first mobility is achieved and when the patient is released into an ambulatory or homecare environment. The CL records the relevant vital signs such as ECG, respiration, pulse oximetry with plethysmogram and movement. The vital data collected with the $C L$ data logger is saved on a memory card for further analysis and is simultaneously transmitted in real-time to a telemedicine server via a smartphone or tablet. The smartphone also provides GPS location information. In addition Cor/log View, an Android ${ }^{\mathrm{TM}}$ Application for viewing recorded vital sign data originating from the $C L$, was developed. CL has also a connector to the generic MedM health cloud. MedM is a generic patient data management system (PDMS) consisting of a cloud portal and a mobile health app. The app runs on Android ${ }^{\mathrm{TM}}$, $i^{\mathrm{TM}}$ and Windows ${ }^{\mathrm{TM}}$. The app can connects wirelessly to the CL physiologic monitor and stores the vital signs in the cloud.
\end{abstract}

\section{Introduction}

During a single treatment path a patient may receive several different vital signs monitors, during a short period $(<24 \mathrm{~h})$, as they move through a hospital system (Figure 1). A patient admitted to the hospital with acute coronary syndrome will be hooked up to different ECG monitors in the ambulance, in the chest pain unit, in the coronary angiography laboratory, in the ICU and on the hospital ward. Furthermore, when the patient is released from the hospital homecare monitoring will be performed with yet another monitoring device. To facilitate medical treatment it would be desirable to have a single wireless patient monitoring device within the hospital and for ambulatory care. For patient safety, especially with the predicted future reduction in healthcare resources (e.g. fewer nurses etc), it is important to improve patient monitoring through technical simplification [1]. On the basis of technical progress in biosignal acquisition and wireless communication [2] the data logger system “Cor/log® BAN BT” was developed. “Cor $/ \log { }^{\circledR}$ BAN BT” allows seamless 24/7 location independent monitoring of relevant vital sign parameters in ambulatory subjects, in home care, and in hospitals.

\section{Methods}

"Cor/log ${ }^{\circledR}$ BAN BT" is a commercial product that has resulted from the German KARDIKOM telemedicine patient monitoring research project. It was the objective of the KARDIKOM patient monitoring research project to prevent secondary life-threatening situations (myocardial infarction, heart failure, arrhythmias) for patients with cardiologic risk constellations by establishing innovative technical and organizational infrastructures to allow continuous 24/7 monitoring of relevant vital signs [3].

Another aim of the project was to certify all components of the wearable wireless vital sign data logger system, the wireless livestream viewer application and a health cloud in accordance with the European Medical Device Directive 93/42/EEC (MDD).

\section{Results}

The wireless data logger system "Cor/log® BAN BT" records the relevant vital signs such as ECG (4 limb leads and 2 precordial leads), respiration (with two belts that measures changes in thoracic and abdominal circumference during respiration), pulse oximetry with 


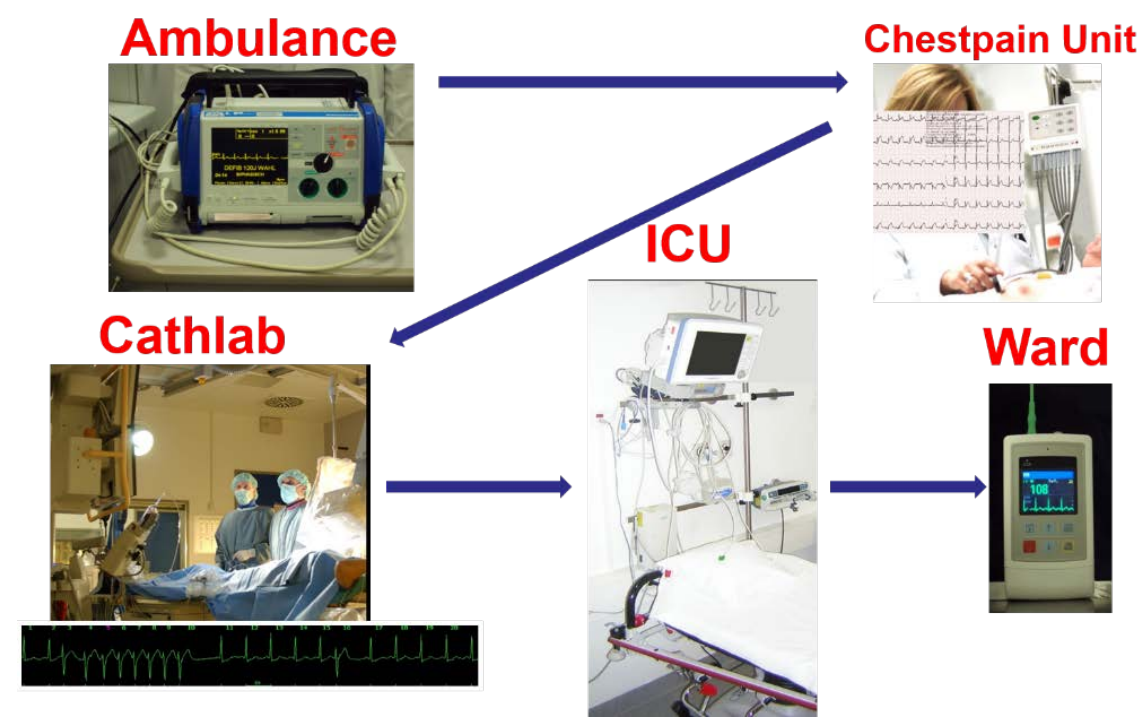

Figure 1: This picture illustrates the different physiological monitors to whom a patient is hooked up within a 24h care cycle for a cardiovascular disease like ACS. Different ECG devices are used on a single treatment path that involves diagnosis on the chest pain unit, treatment in the cardiac catheterization lab and monitoring on the intensive care unit (ICU) and the hospital ward.

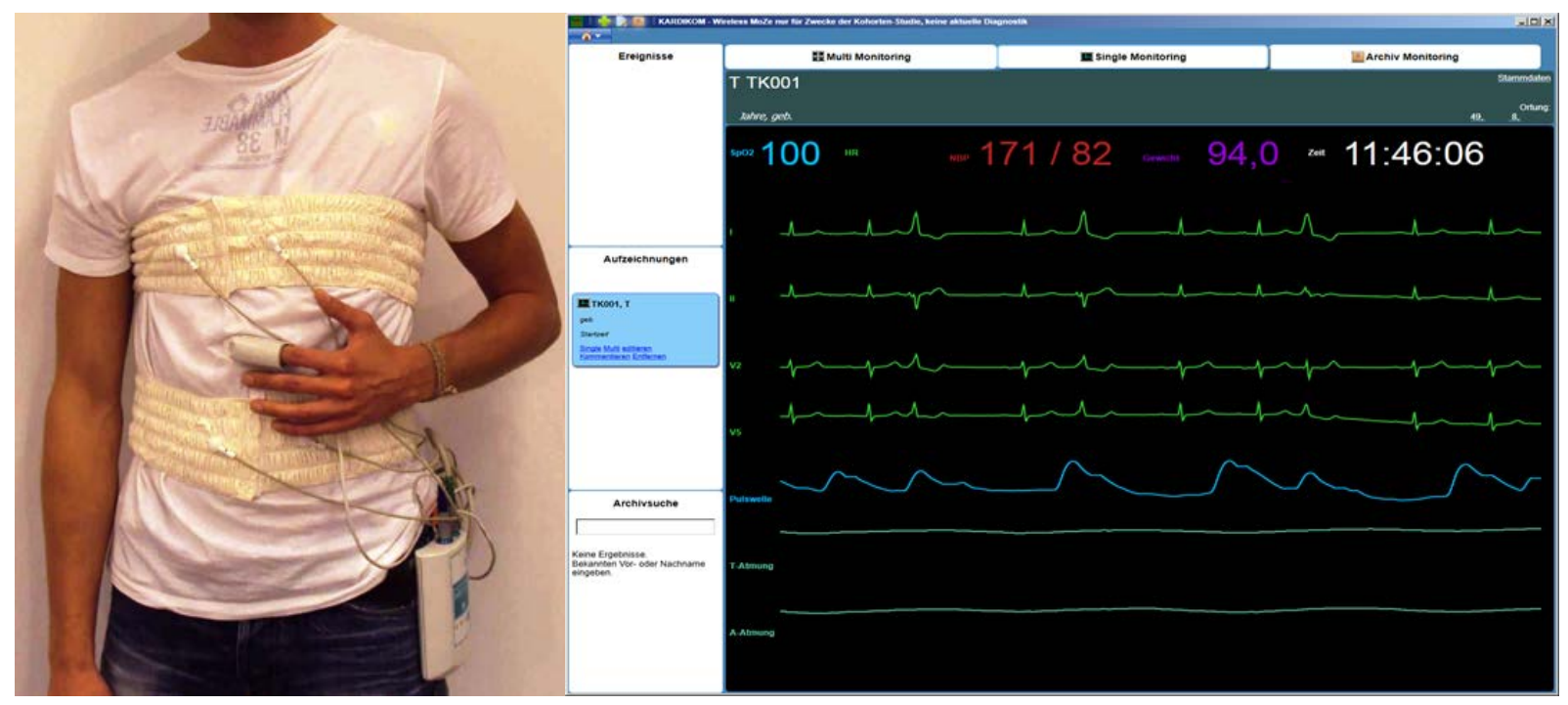

Figure 2a \& b: The left picture illustrates the application of the wireless data logger system "Cor/log® BAN BT". The person carries the "Cor/log® BAN BT" on his left hip. The following sensors are visible: the two breathing belts, one around the thorax and one around the abdomen and the pulse oximeter on the left index finger. The ECG electrodes are attached underneath the shirt. Figure 2b:The right picture is a screen shot of the almost realtime telemedical monitoring software. The software displays the following physiological parameters in numbers: $\mathrm{SpO}_{2}$ in blue; heart rate in green; blood pressure in red, and weight in purple (pressure and weight from external devices). The following physiological vital sign waveforms displayed from top to bottom are: ECG in green, $\mathrm{SpO}_{2}$ photoplethysmogram in blue and two respiration belt waves in cyan.

plethysmogram and movement (via an integrated 3D acceleration sensor), see Figure 2a. The physiological vital sign data collected with the "Cor/log® BAN BT" data logger is saved on an integrated memory card for further offline analysis and is simultaneously transmitted as a telemetry Bluetooth ${ }^{\circledR}$ livestream to a smartphone, a tablet or a home gateway device. Three different options for further biosignal monitoring are then available:

a) A dedicated mobile app that forwards the biosignals and the GPS location information to a dedicated 
telemedicine server (Figure 2b).

b) A monitoring application for livestream viewing and retrieving of the recorded vital sign data on a tablet (Figure 3).

c) A connectivity app to the generic MedM health cloud platform. MedM is a generic patient data management system (PDMS) for remote patient monitoring consisting of a mobile health app, a cloud portal, cloud services and a set of EMR/EHR integration APIs. The app runs on Android, iOS ${ }^{\mathrm{TM}}$ and Windows $^{\mathrm{TM}}$ (Figures 4,5,6). The app establishes a wireless connection to the $\mathrm{Cor} / \log { }^{\circledR}$ BAN BT physiologic monitor and stores the vital signs (ECG and $\mathrm{SpO}_{2}$ ) in the cloud. The generic MedM app is compatible with more than 200 medical sensors from 50 vendors. MedM allows anywhere and anytime web-based access to patient health information and features historic and almost realtime data views, trend graphs, thresholds \& notifications and reports.

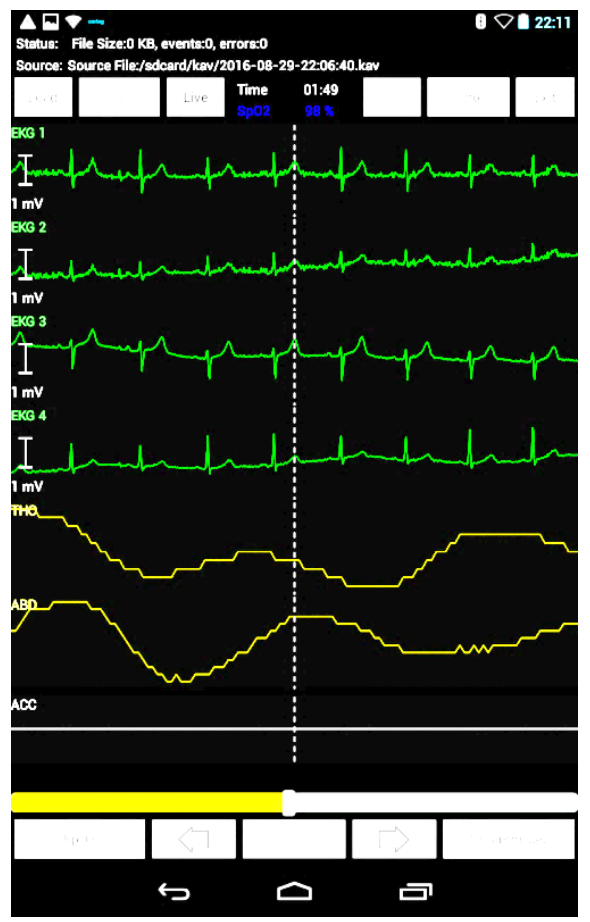

Figure 3: Screenshot of. Cor/log ${ }^{\circledR}$ View Application for viewing and retrieving the bluetooth livestream from the Cor/log® BAN BT patient monitor. ECG waveforms in green, respiration waveforms in yellow.

In the KARDIKOM application study the data logger was tested on 134 subjects within the hospital and in an out-patient homecare or work environment. The application study found that the wireless $24 / 7$ vital sign data logger system is suitable for continuous monitoring within the hospital or in out-patient telemedicine settings. The detailed study results can be found in the
KARDIKOM application study report [4].

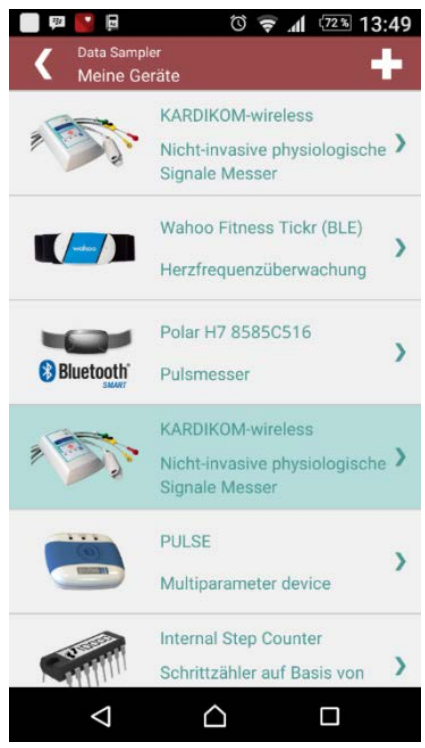

Figure 4: Screenshot of the MedM heath app on an android device. The app connects to more than 200 medical sensors by 50 vendors, displays the measured parameter on the smartphone and forwards the data for further storage to a cloud server. The screenshot displays the devices selected by the user for measurements with their smartphone and a user with the name "Data Sampler”.

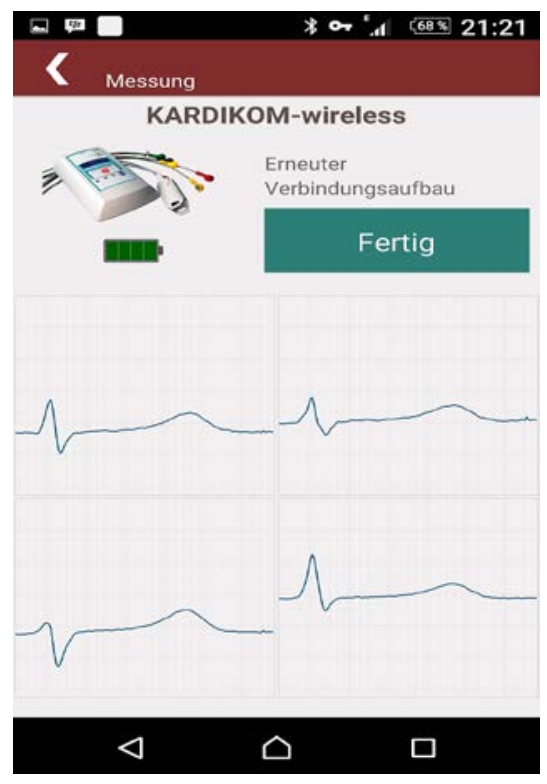

Figure 5: Screenshot of the MedM app with the bluetooth ECG (4 channels) livestream from the Cor/log ${ }^{\circledR}$ BAN BT vital sign data logger. 


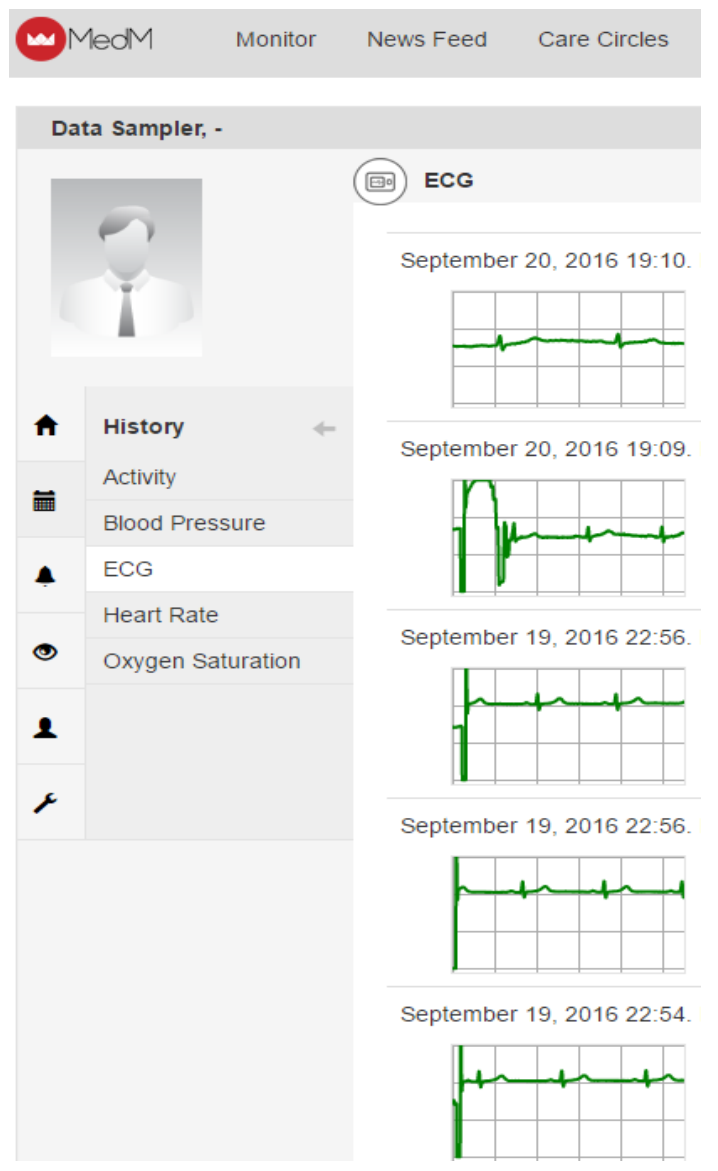

Figure 6) Screenshot of the MedM web-based Patient Health Information. The page does list a report history of Cor/log ${ }^{\circledR}$ BAN BT ECG recordings.

At the time of writing the medical regulatory compliance of the monitoring units is as following:

Depending on intended use "Cor/log ${ }^{\circledR} B A N B T$ " and "Cor/log View" are cleared as Class I products (intended use: patient data management system (PDMS)) or as class IIa medical devices (intended use: patient vital data recorder) in accordance with the annex VII of the European Medical Device Directive 93/42/EEC.

The MedM Remote Patient Monitoring System is cleared as a Class I PDMS according to the EU Medical Device Directive in Europe and cleared in the US as Medical Device Data System (MDDS) according to FDA Regulations and thus is exempt from FDA 510(k) process.

\section{Discussion and conclusions}

Continues 24/7 mobile physiological multi-parameter monitoring is possible with small data loggers. Cor $/ \log { }^{\circledR}$ BAN BT is suitable for general use by individuals/patients and caregivers for monitoring in hospitals but also in homecare settings. Cor/log ${ }^{\circledR}$ BAN
BT does not claim the status of a final 2016 product design, but it is a complete design and covers all relevant regulatory aspects. It is mandatory to certify the devices and all software according the medical device directives [5]. However, the journey from a prototype device to full medical certification is both resource and cost intensive. Advances in technology for miniaturization, and battery longevity; the technical findings of the application study and suggestions of the users will have important impact for further system improvements. But any improvement does require a very expensive medical device certification. In the future smartphone based patient monitoring are increasingly entering the field of patient monitoring and they seem not to be inferior to conventional monitoring technologies [6].

\section{References}

[1] Drews, F.A. (2008). Patient Monitors in Critical Care: Lessons for Improvement. In: Advances in Patient Safety: From Research to Implementation. Volume 5, AHRQ Publication Nos. 050021 (1-5). Agency for Healthcare Research and Quality, Rockville, MD. 2008.

[2] Kennedy HL. The evolution of ambulatory ECG monitoring. Prog Cardiovasc Dis. 2013 Sep-Oct;56(2):12732.

[3] Hilbel T, Feilner S, Zugck C, Struck M, Hofmann C, Heinig A, Giannitsis E, Katus A. Application study for a vital-sensor network applied to seamless monitoring of patients with acute cardiac risk constellation in in-patient and out-patient settings. KARDIKOM-Wireless. Journal of Electrocardiology 2014, Volume 47, Issue 6, 912.

[4] Hilbel T, Feilner S. Machbarkeitsstudie für ein kardiologisches Vital-Sensorik Netz für ein engmaschiges kontinuierliches Monitoring von Patienten mit akuter kardialer Risikokonstellation im stationtären und außerklinischen Umfeld, Short title: KARDIKOMWireless. Technische Informationsbibliothek u. Universitätsbibliothek Hannover, Report [online] 2013. [cited 2016 Sept 21]. Available from: URL: edok01.tib.uni-hannover.de/edoks/e01fb14/788065416.pdf.

[5] FDA, Mobile Medical Applications, Guidance for Industry and Food and Drug Administration Staff [Online]. 2015. [cited 2016 Aug 22]. Available from: URL:http://www.fda.gov/downloads/MedicalDevices/Devi ceRegulationandGuidance/GuidanceDocuments/UCM2633 66.pdf.

[6] Narasimha D, Hanna N, Beck H, Chaskes M, Glover R, Gatewood R, Gudleski G, Danzer S, Curtis AB. A smart phone-based ECG recorder is noninferior to an ambulatory event monitor for diagnosis of palpitations. Heart Rhythm, Vol. 13, Issue 5, May Supplement 2016: S149:P001-132.

Address for correspondence.

Thomas Hilbel MD

Heidelberg University Hospital, Department of Cardiology

Im Neuenheimer Feld 410

69120 Heidelberg, Germany

E-mail:thomas.hilbel@med.uni-heidelberg.de 